\title{
Subject Index Vol. 22, 1995
}

\section{Acquired aqueductal stenosis}

309 Acrosyndactyly 29 Aneurysm, traumatic intracra-

nial 15 Angio-osteohypertrophy

syndrome 328 Astrocytoma 281 Atypical teratoid tumor 214

Birth defects 29 Blink reflex 120 Bone 241 Brain 189

- tumor(s) $1,74,274,289$

- -, pediatric 8 Brainstem auditory evoked

potential 120

- dysfunction 120

Canavan disease 214 Cancer 189 Caudal regression 20 Central nervous system 214 Cerebral neuroblastoma 1

- palsy 255 Cerebrospinalfluid 111

- seeding 210

- - shunt(s) 101, 132 Cervical spine 147 Chemotherapy 1, 189 Chiari malformation 251

- type-I malformation 299

type-II malformation 120 Children 281

Choroid plexus carcinoma 189 Complications 309 Computed tomographic scan

315 Congenital anomalies 303 Continence 248 Cord motion 115 Corpus callosum agenesis 321 Cortical dysplasia 181 Cost analysis 255 Cranial suture 162 Craniocervical junction 88 Craniofacial surgery 57 Craniopharyngioma 210,251 Craniosynostoses 29, 162,235,

241,270 CTscan 74,141 Cytogenetics 204

Dandy-Walker malformation

309 Double-compartment hydro-

cephalus 309 Dystopia 57

Editor's responsibilities 223 EEG 174

Electrocorticography 181 Epilepsy 167, 174 -, intractable 181

Fourth ventricle 309 Fracture 101

Gadolinium 8 Ganglioglioma 181 Glioma 65,281

Hamartoblastoma 303 Hamartoma 303 Head injury 81, 108 Hemangiopericytoma 274 Hemihypertrophy 328 Hydrocephalus 29,132,141, 228,315,328 Hydromyelia 251,299 Hydrosyringomyelia 251 Hypothalamus 303

${ }^{125}$ I 289

In situ hybridization 204 Instability 147 Internal carotid artery occlusion 108 Interstitial implants 289 Intracranial pressure 235 Intradural arteriovenous fistula,

pediatric 158 Intramedullary spinal cord

abscess 88 Intraoperative anaphylaxis 96 Intrathecal baclofen 255 Intraventricular hemorrhage

315 Invasive monitoring 174 Isolated fourth ventricle 309

Klippel-Trenaunay-Weber syndrome 328

Lactate 81 Lambdoid 162 Latex allergy 96 Lesionectomy 167

Lipomyelomeningocele 20,137 Lower extremity pain 248

Macrocephaly 228 Magnetic resonance angiog-

raphy 108 - - imaging 20, 120,299 Manuscript review 223 Medial canthus 57 Medulloblastoma 197 Metalloproteases 204 Midbrain 65 MRI 167 Myelinoclastic diffuse sclerosis 266 Myelomeningocele 96, 120,

132 Myelopathy 158

Nasal encephalocele 57 Nasolacrimal apparatus 57 Neoplasm 65 Neurenteric cysts 88 Neuroaxonal dystrophy 321 Neuronal storage 321

Occult spinal dysraphism 20 Oligodendroglioma 281 Orbital anatomy 57 Osteoblasts 241 Osteoclasts 241 Osteopetrosis 321 Outcome 115

Pallister-Hall syndrome 303

Papilledema 270

Pediatric tumors 289

Pediatrics 147

Periorbita 57

Peritoneal catheter 101

Pfeiffer's syndrome 270

Phase MRI 115

Plagiocephaly 162,235

Polydactyly 303 
Primitive neuroectodermal tumor 1

Protein kinase C 281

Radiation therapy 1 Radiosurgery 197 Radiotherapy 197 Recurrence, tumor 181

Recurrent tumor 210 Reduction cranioplasty 228 Residual tumor 74 Rhabdoid tumor 214

- -, invasion 204

Risks 228

Sacral agenesis 20 Scaphocephaly 235 Schilder's disease 266 Screw fixation 147 Seizure outcome 181 Selective posterior rhizotomy 255 Shunt(s) 141,309

- complications 132

revision 315 Shunting complications 309 Signal transduction 281 Skull base surgery 57 Somatosensory evoked potential 120 Spasticity 255 Spectroscopy 81 Spinal cord 158

- tumours 88

- dysraphism 137

- lipoma 111

- tethering 137

Split notochord syndrome 88 Surgical technique 228 Sutural fusion 241 Syringomyelia 248,299

Tamoxifen 281 Tectal region 65 Telecanthus 57 Tethered cord 115

- spinal cord 111

Thallium 8

Timing of surgery 137

Tissue inhibitor, metallopro

teases 204

Tonsillar herniation 251 Trapped fourth ventricle 309 Trigonocephaly 235 Tumor-associated epilepsy 181 Turner’s syndrome 266

Visual acuity 270 